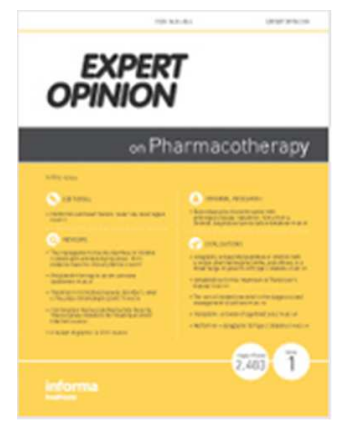

Please download and read the instructions before proceeding to the peer review

\title{
Pirfenidone for the treatment of idiopathic pulmonary fibrosis
}

\begin{tabular}{|r|l|}
\hline Journal: & Expert Opinion On Pharmacotherapy \\
\hline Manuscript ID: & EOOP-2013-0267.R1 \\
\hline Manuscript Type: & Drug Evaluation \\
\hline Keywords: & Antifibrotic drugs, Idiopathic pulmonary fibrosis, Pirfenidone, Treatment \\
\hline \multicolumn{2}{|r}{} \\
\hline
\end{tabular}

SCHOLARONE ${ }^{\mathrm{m}}$

Manuscripts 


\section{PIRFENIDONE FOR THE TREATMENT OF IDIOPATHIC PULMONARY FIBROSIS}




\begin{abstract}
Introduction. Idiopathic pulmonary fibrosis (IPF) is a chronic, progressive, and lethal fibrosing interstitial pneumonia. The median survival from the onset of the symptoms is $2.8-4.2$ years and the 5 -year survival rate is $20 \%$. Its poor prognosis, combined with the scarcity of treatment options, provides a strong rationale for the development of novel therapeutic strategies. During the last decade there has been a huge rise in clinical trials with antifibrotic drugs, although only pirfenidone has shown a beneficial effect.
\end{abstract}

Areas covered. This article reviews the medical literature on the effectiveness and safety of pirfenidone in IPF, by means of a PubMed search from 1995 to present, completed by some data on file from the manufacturer.

Expert opinion. Pirfenidone is the only antifibrotic drug approved for the treatment of IPF. Pirfenidone presents a meaningful clinical effect on reductions in the decrease in forced vital capacity (FVC), six minute walk test (6MWT) distance and mortality, and it improves the progression-free survival in IPF patients with mild-moderate disease. Pirfenidone is well tolerated, with the most common side-effects being gastrointestinal discomfort and photosensitivity. Pirfenidone has a favorable benefit-risk profile and represents a suitable treatment option for patients with mild-moderate IPF.

Key words. Antifibrotic drugs, Idiopathic Pulmonary Fibrosis, Pirfenidone 


\section{INTRODUCTION}

IPF is a chronic, progressive, and lethal fibrosing interstitial pneumonia, limited to the lung and associated with the histopathological and/or radiological pattern of usual interstitial pneumonia (1). Although its etiology is unknown and the pathogenesis only partly understood, our current knowledge suggests that damage to the alveolar epithelium is probably an important early event followed by an aberrant healing response. The median age at diagnosis is 66 years, with a male predominance, and prevalence has been increasing in recent years. The incidence of IPF is estimated to be between 4.6 and 7.4 cases / 100000, and the prevalence is between 13 cases/100000 for females and 20 cases/100000 for males (1). IPF is characterized by a progressive decline in pulmonary function, which quickly leads to respiratory failure and death, although some patients may have episodes of acute respiratory worsening despite previous stability. With a median survival from the onset of the symptoms of 2.8-4.2 years and a 5 -year survival rate approaching $20 \%$, IPF is more lethal than many malignant diseases. The poor prognosis, combined with the scarcity of treatment options, provides a strong rationale for the development of novel therapeutic strategies for this disease. During the last decade there has been a huge rise in clinical trials with antifibrotic drugs. In phase III randomized clinical trials with interferon gamma 1-b, pirfenidone, macitentan, bosentan, ambrisentan, warfarin, triple therapy (n-acetylcisteine, glucocorticoids, azathitoprine), etanercept and sildenafil, only pirfenidone has shown any beneficial effects in patients with IPF (2-4). Several national guidelines on IPF diagnosis and treatment from Spain, Germany, Denmark, Sweden, Austria, and Ireland, have recently recommended pirfenidone as first-choice therapeutic 
agent in IPF patients with mild-moderate disease, defined as FVC predicted > $50 \%$ (5-9). The National Institute for Health and Clinical Excellence (NICE) recommends pirfenidone as a therapeutic option in IPF patients with FVC predicted between 50 and $80 \%$. (10).

\section{OVERVIEW OF THE MARKET}

Pirfenidone is the only pharmacological agent approved for the treatment of mild-moderate IPF (11). Nowadays, there is no drug available for the treatment of patients with IPF with advanced disease.

\section{INTRODUCTION TO PIRFENIDONE}

Pirfenidone is a pleiotropic molecule with antifibrotic, anti-inflammatory and antioxidant effects (12). The antioxidant effect occurs through its ability to scavenge reactive oxygen species (13). Studies in animal models of pulmonary fibrosis have shown that pirfenidone attenuates a range of inflammatory and profibrotic molecules while downregulating histological markers of fibrosis and cellular proliferation (14). Pirfenidone inhibits the expression of transforming growth factor (TGF)- $\beta$ and the tissue inhibitor of metalloproteinase-1 (TIMP-1), and blocks the proliferative effects of platelet-derived growth factor (PDGF). Furthermore, pirfenidone inhibits the release of proinflammatory cytokines such as, tumor necrosis factor (TNF)- $\alpha$, interleukin (IL-1)- $\beta$, IL-6, IL-8 and IL-12, as well increasing the expression of the anti-inflammatory cytokine IL-10, attenuating the release of chemotactic cytokines and reducing the accumulation of inflammatory cells in response to different stimuli (14-19) 
In addition to the modulation of mediators in animal models, cell-based assays have demonstrated that pirfenidone inhibits the expression of heat shock protein (HSP) 47 in cultured normal lung fibroblasts stimulated with TGF- $\beta-1$; it was also able to inhibit the expression of collagen in fibroblasts isolated from IPF patients $(20,21)$. Moreover, pirfenidone inhibits the overexpression of collagen type I and HSP47 in the alveolar epithelial cell line (A549 cells), (22).

\subsection{CHEMISTRY}

Pirfenidone is an orally bioavailable synthetic molecule. Pirfenidone is a heterocyclic pyridine (5-methyl-1-phenyl-2-[1H]-pyridine) with a molecular weight of 185.22. Pyridines are derived from coal combustion, and can be synthesized from aldehyde and ammonia. They are highly soluble in dimethylsulfoxide, twice in water and chloroform, and in alcohol. In aqueous solution the maximum possible concentration is $2 \%$. (23).

\subsection{PHARMACOKINETICS AND METABOLISM}

Oral pirfenidone has linear pharmacokinetics over the dose range of $200-600$ mg. (24). After a single dose of pirfenidone with food at the recommended maintenance dose of $801 \mathrm{mg}$, the mean maximum plasma concentration $\left(\mathrm{C}_{\max }\right)$ is $7.9 \mathrm{mg} / \mathrm{L}$ in healthy adults. Absorption occurs quickly, the time to maximun ( $\left.T_{\max }\right)$ values being achieve in $30-60 \min (25)$. Median time to $C_{\max }$ is $3.5 \mathrm{~h}$. However, the $C_{\max }$ is significantly lower when the drug is administered in the fed versus fasting states (26). It has been shown that healthy volunteers who received pirfenidone with food had a lower incidence of gastrointestinal adverse events, than those who received pirfenidone without food. In clinical trials, at 
concentrations of $1-1000 \mathrm{mg} / \mathrm{L}, 50-58 \%$ of pirfenidone bound to plasma proteins, predominantly albumin (27). Pirfenidone is predominantly metabolized by the cytochrome (CYP) P450 CYP1A2 enzyme, although other CYP enzymes, including CYP2C9, 2C19, 2D6, and $2 \mathrm{E}$, also contribute to the metabolism. The major metabolite of pirfenidone is 5-carboxy-pirfenidone which is inactive. After a single $801 \mathrm{mg}$ dose, the terminal elimination half life was 2.9 $\mathrm{h}$ (without food) and $2.4 \mathrm{~h}$ (with food). No significant gender differences were noted for the pharmacokinetic variables (26). Eighty percent of the administered dose was excreted in the urine primarily as 5-carboxy-pirfenidone and less than $1 \%$ of the dose recovered in the urine was unaltered, after $6 \mathrm{~h}$ of administration. Pirfenidone bioavailability is increased by a mean of $60 \%$ in patients with moderate hepatic function impairment. No clinical relevant changes have been observed in pharmacokinetics in patients with mild to severe renal function impairment. However, the drug is contraindicated in patients with severe renal impairment (creatinine clearance of $<30 \mathrm{~mL} / \mathrm{min}$ ) or end stage renal disease requiring dialysis. (28)

Pirfenidone may interact with drugs that inhibit CYP1A2, particularly fluvoxamine, ciprofloxacin, and propafenone. Consumption of grapefruit also inhibits CYP1A2. Concomitant treatment with inhibitors of other CYP isoenzymes involved in the metabolism of pirfenidone (fluconazole, chloramfenicol, fluoxetine, paroxetine, amiodarone) may interact with pirfenidone. Pirfenidone can also interact with CYP1A2, inducers, such as tobacco smoke and omeprazole (27) (Table 1).

\section{CLINICAL EFFICACY}




\subsection{Clinical trials}

In a prospective, open-label phase II study, pirfenidone was evaluated for its tolerability and usefulness in patients with advanced IPF, progressive disease and lack of response to conventional therapy (prednisone with or without immunosuppressives). Fifty-four patients were followed for mortality, changes in lung function and adverse effects. Patients whose lung function had deteriorate before enrolement appeared to stabilize after beginning treatment. Patients with higher single-breath diffusing capacity for carbon monoxide ( $\left.D L_{c o}\right)(>30 \%$ predicted) at entry had longer survival. The adverse effects were minor (29).

The first large-scale trial of pirfenidone was a Japanese multicenter randomized placebo-controlled phase II study of 107 subjects who received either pirfenidone $600 \mathrm{mg}$ three times daily $(n=72)$ or placebo $(n=35)$. The trial was designed to run for 1 year. Although there was a significant decrease in the decline of FVC in the pirfenidone group, 9-month interim results showed that five subjects from the placebo group had suffered acute exacerbation of the disease compared to none in the pirfenidone group, and the study was halted (30).

A phase III clinical trial conducted over 52 weeks was also developed in Japan. A total of 275 patients were randomized to either high-dose (1800 mg/day) or low-dose (1200 mg/day) pirfenidone or placebo the ratio of $2: 1: 2$. Significant differences were observed in vital capacity (VC) decline (primary end-point) between the placebo group and the high-dose group and improved progressionfree survival time was seen in the high-dose group. (31). To find out which patients specifically benefit from pirfenidone, an additional exploratory analysis was performed with the data of this trial. Significant efficacy of pirfenidone in 
reducing the decline in VC was seen in a subpopulation with $\% \mathrm{VC} \geq 70 \%$ and oxygen saturation at baseline $\left(\mathrm{SpO}_{2}\right)<90 \%$. These are mutually conflicting characteristics. According to the author opinion, the desaturation exhibited by the subgroup with the better-perserved lung function may have been due to the develipment of fibrosis with inflammatory edema and not to established fibrosis. In this subpopulation, pirfenidone also suppressed cough and dyspnea (32). Recently, marginal decline in FVC $(5 \%)$ has been reported as being associated with poor outcome in IPF. In the same clinical trial, Taniguchi et al sought to evaluate the efficacy of pirfenidone from the aspects of $5 \%$ changes in VC. When $5 \%$ change in VC was used as an index instead of the $10 \%$ change, the efficacy of pirfenidone could be evaluated with higher sensitivity over the 12 months study. (33).

The CAPACITY phase III trials consisted of two concurrent multinational randomized double-blind placebo-controlled phase III trials (004 and 006) conducted over 72 weeks and designed to evaluate the safety and efficacy of pirfenidone in patients with mild-moderate disease $(34,35)$. In trial 004, 174 patients were assigned to high-dose pirfenidona (2403 mg/day), 87 to low dose (1197 mg/day) and 174 to placebo. In study 006, 171 patients were assigned to high-dose pirfenidone (2403 mg/day), and 173 to placebo. In study 004, the higher dose of pirfenidone met the primary endpoint, decreasing the decline in FVC. In contrast, trial 006 failed to show a significant reduction in FVC, although there was a reduced decline in distance walked in the 6MWT (secondary endpoint). The differences observed between the two studies in the effect of pirfenidone may be explained by the fact that the subjects in the 006 placebo group had a slower rate of decline compared to those in 004 . When 004 and 
006 study data were pooled, pirfenidone shows meaningful clinical effects on FVC $\%$ predicted (decrease in the decline by $30 \%$ ), progression free survival and 6MWT distance at the dose of $2403 \mathrm{mg} /$ day.. Although these trials were not powered to detect an effect on mortality, IPF-related mortality was reduced by 28\%. A Cochrane review encompassing the two Japanese trials and the CAPACITY studies, has demonstrated that pirfenidone improves progressionfree survival by $30 \%$. (36).

The RECAP study is an ongoing open-label extension study evaluating the long-term administration of pirfenidone in patients who completed phase III CAPACITY program. Preliminary results in 178 patients show that FVC and survival outcomes in patients newly treated with pirfenidone in the RECAP study were similar to those treated in the CAPACITY trials. These data provide further evidence to support the beneficial effect of pirfenidone in IPF (37). A phase III study comparing pirfenidone with placebo in patients with mild-tomoderate IPF is currently under way in the US (ASCEND trial, NCT01366299). The PANORAMA study (A Randomized, Double-Blind, Placebo-Controlled, Phase II Study of the Safety and Tolerability of $N$-Acetylcysteine in Patients with Idiopathic Pulmonary Fibrosis with Background Treatment of Pirfenidone, EUDRACT 2012-000564-14) is also ongoing.

\subsection{Posology and method of administration}

Pirfenidone is indicated in adults for the treatment of mild-to-moderate IPF (FVC $>50 \%$ predicted (5-9). The National Institute for Health and Clinical Excellence recommends pirfenidone in patients with FVC between $50 \%$ and $80 \%$ predicted (10). The approved dose is $2403 \mathrm{mg} /$ day. Each capsule contains $267 \mathrm{mg}$. Upon 
initiating treatment, the dose should be titrated to the recommended daily dose over a 14-day period: days 1 to 7 , one capsule three times a day (801 mg/day); days 8 to 14, two capsules, three times a day (1602 mg/day) and day 15 onward, three capsules, three times a day (2403 mg/day). The drug should be taken with food to reduce the possibility of gastrointestinal adverse events and dizziness (27). The recommended treatment duration is at least 12 months. If there is improvement or stabilization of the disease, the treatment should be continued. In case of deterioration, the advisability of continuing treatment or instigating other therapeutic strategies should be considered for each patient. However, it is recommended that treatment should be discontinued if there is evidence of disease progression, that is, a decline in per cent predicted FVC of $10 \%$ or more within any 12 month period $(5,10)$.

\subsection{Post marketing studies}

Open-label studies have been developed, to confirm the efficacy, safety and tolerability of pirfenidone and to optimize its indications.

A European Named Patient Program (NPP) was established by Intermune ${ }^{\circledR}$ to make pirfenidone accessible to patients with IPF during the period between the authorization of the drug and its availability upon prescription. The results of this program have not yet been published. The PASSPORT study (PostAuthorisation Safety Study of Esbriet ${ }^{\circledR}$ (Pirfenidone): A Prospective Observational Registry to Evaluate Long-Term Safety in a Real-World Setting) is also ongoing; its objective is to evaluate the long-term safety of pirfenidone. In a retrospective study, Okuda et al (38) have described the effects of pirfenidone, at the dose of $1800 \mathrm{mg} /$ day during 6 months, in 76 patients with 
mild-to-severe IPF. Pirfenidone tended to attenuate the degree of decline in FVC, particularly in the subgroup of patients with FVC $<60 \%$ and showed greater efficacy in patients with decline in FVC $>150 \mathrm{ml}$ during the 6-months period before the start of therapy. Thus, the degree of disease progression prior to the initiation of therapy had an impact on the response to pirfenidone. The most frequent adverse effects were anorexia (42\%), increase in gamma glutamyl transpeptidase (22\%), fatigue (14\%) and photosensitivity (18\%). All the reported adverse effects were, howevver, mild, reversible and left no sequelae. Moreover, Bonella et al. (39), have reported data on the safety and efficacy of pirfenidone $(2403 \mathrm{mg} /$ day) in 45 patients with mild-moderate IPF (mean duration of treatment 48 weeks). Sixteen patients (35\%) received pirfenidone as monotherapy and $29(65 \%)$ in combination with corticosteroids and /or $\mathrm{N}$ acetylcisteine. The course of the disease was stable during treatment with pirfenidone in two out of every three patients. Twenty-six patients (58\%) suffered from side effects, mostly gastrointestinal, but pirfenidone was discontinued because of side effects in only six patients. The results of this study confirm the efficacy and safety of pirfenidone in the treatment of IPF.

Iwasawa et al. (40) investigated the usefulness of high resolution CT scan (HRCT) in the imaging assessment of the response to pirfenidone therapy. Seventy-eight patients (38 treated with pirfenidone and 40 matched controls) were given HRCT on two occasions, with a one-year interval in between. A significantly larger proportion of patients treated with pirfenidone showed more stable pulmonary function parameters than the controls (65.6\% vs $37.5 \%)$. The decline in VC correlated with the increase in fibrotic lesions in CT scan. These 
results suggest that CT scan may be useful for evaluating the pirfenidoneinduced slowing progression of pulmonary fibrosis.

\section{SAFETY AND TOLERABILITY}

Jiang et al (41) have reported the results of a meta-analysis to analyze the safety profile of pirfenidone for treating pulmonary fibrosis. Six-randomized controlled trials were analyzed. A total of 1073 patients were enrolled, 561 in the pirfenidone group, and 512 in the placebo group. Four trials assessed the treatment of IPF and the remaining two studies, assessed the treatment of Hermansky-Pudlak syndrome $(30,31,34,42,43)$. The combined results of the six trials revealed that the pirfenidone group had a significantly higher rate of gastrointestinal, neurological and dermatological adverse events. However, adverse effects were generally mild or moderate in severity and without any clinically significant consequences. Most of the adverse effects disappeared with a decrease in the dose or temporary discontinuation of medication.

The most commonly reported $(\geq 10 \%)$ adverse reactions during treatment with pirfenidone at a dose of $2403 \mathrm{mg} /$ day are nausea, rash, fatigue, diarrhea, dyspepsia and photosensitivity reaction (table 2). Less frequent adverse effects are anorexia, insomnia, hot flush and alteration in the hepatic enzymes alanin and aspartate aminotransferases, and gamma glutamyl transferase. (27).

Gastrointestinal events are less frequent if the drug is administered with food. Patients with photosensitivity reaction or rash should use protective sun creams, to wear clothing that protects against sun exposure and avoid direct sunlight. In case of adverse events, the dose of pirfenidone should be adjusted 
or the treatment discontinued, according to the established recommendations. There are no data on the use of pirfenidone during pregnacy. (27).

Pirfenidone is contraindicated in case of hypersensitivity to the drug or to any of its excipients, concomitant use of fluvoxamine, severe hepatic impairment or end-stage liver disease or severe renal impairment or end-stage renal disease requiring dialysis. Pirfenidone should be used with caution in patients treated with inhibitors or inducers of CYP1A2 (table 1) (27).

\section{REGULATORY AFFAIRS}

Pifenidone is an orally administered pyridine that was granted orphan drug approval in the European Union for the treatment of mild-to-moderate IPF in 2011 and it is the only pharmacological agent available for this indication (44). In Europe, it is marketed under the brand name Esbriet ${ }^{\circledR}$ by Intermune Inc. In Japan, pirfenidone has been approved for marketing since 2008 under the brand name Pirespa ${ }^{\circledR}$ by Shionogi \& Co. In 2010, the Food and Drug Administration (FDA) denied approval for pirfenidone. A new drug application to the FDA is expected depending on the results of the ongoing phase III trial ASCEND (Efficacy and safety of Pirfenidone in Patients with Idiopathic Pulmonary Fibrosis).

\section{CONCLUSION}

IPF is a chronic, progressive, and lethal fibrosing interstitial pneumonia. Its poor prognosis, combined with the scarcity of treatment options, provides a strong rationale for the development of novel therapeutic strategies for this disease. During the last decade several clinical trials with antifibrotic drugs have been 
developed, although only pirfenidone has shown a beneficial effect. Recent national guidelines and the National Institute for Health and Clinical Excellence recommend pirfenidone as first-choice therapeutic agent in IPF patients with mild-moderate disease, defined as FVC $>50 \%$ of predicted. Pirfenidone's adverse-effect profile is acceptable in a disease as severe as IPF. Nowadays, pirfenidone is the only pharmacological agent approved for the treatment of mild-moderate IPF.

\section{EXPERT OPINION}

IPF is a progressively fibrotic disease with a median survival from the onset of the symptoms of 2.8 to 4.2 years. The search for effective treatment has involved numerous clinical trials with potentially antifibrotic drugs, although only pirfenidone has shown any beneficial effect. Four key clinical trials have endorsed the efficacy and tolerability of pirfenidone. Pirfenidone has shown clinically meaningful effects on decreases in the decline of \% FVC, 6MWT distance and mortality, as well as on improvements in progression-free survival. In addition, pirfenidone could prevent symptoms related to disease progression, decrease dependency on oxygen, increase survival and reduce hospitalizations. Pirfenidone is well tolerated, with the most common side-effects being gastrointestinal discomfort and photosensitivity. Open-label and placebocontrolled studies are underway to confirm the efficacy and long-term effects of pirfenidone. Recently published national guidelines recommend pirfenidone as a first-choice therapeutic agent in IPF patients with mild-moderate disease. Pirfenidone has a favorable benefit-risk profile and represents a suitable treatment option for IPF. 
1

2

3

4

5

6

7

8

9

10

11

12

13

14

15

16

17

18

19

20

21

22

23

24

25

26

27

28

29

30

31

32

33

34

35

36

37

38

39

40

41

42

43

44

45

46

47

48

49

50

51

52

53

54

55

56

57

58

59

60

\section{DRUG SUMMARY BOX}

Drug name

Pirfenidone

Phase

Marketed

Indication

mild-moderate idiopathic pulmonary fibrosis

Pharmacology description

antifibrotic, anti-inflammatory, antioxidant

Route of administration

oral

Chemical structure

Pivotal trials

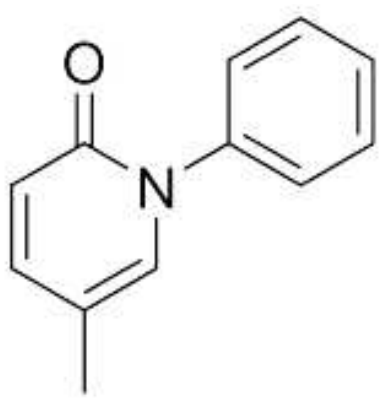

$(30,31,34)$ 


\section{REFERENCES}

1. Raghu G, Collard HR, Egan JJ et al. An official ATS/ERS/ALAT statement: idiopathic pulmonary fibrosis: evidence-based guidelines for diagnosis and management. Am J Respir Crit Care Med 2011; 183: 788824

2. Luppi $F$, Spagnolo $P$, Cerri $S$ et al. The big clinical trials in idiopathic pulmonary fibrosis. Curr Opin Pulm Med 2012; 18: 428-432

3. Raghu G, Behr J, Brown KK et al. Treatment of idiopathic pulmonary fibrosis with ambrisentan: a parallel randomised trial. Ann Intern Med 2013; 158: 641-649

4. Raghu G, Million-Rousseau R, Morganti A et al. Macitentan for the treatment of IPF: the randomized controlled MUSIC trial. Eur Respir J 2013; May 16 (Epub ahead of print)

5. Xaubet A, Ancochea J, Bollo E et al. Guidelines for the diagnosis and treatment of idiopathic pulmonary fibrosis. Arch Bronconeumol 2013; 49: 343-353

6. Behr J, Günther A, Ammenwerth $W$ et al. German Guideline for Diagnosis and Management of idiopathic Pulmonary Fibrosis. Pneumologie 2013; 67:81-111 
7. Idiopathic pulmonary fibrosis guidelines. Danish Society of Respiratory Medicine www.lungemedicin.dk (Last accesed 29 August 2013).

8. Position Statement from the Irish Thoracic Society on the treatment of idiopathic pulmonary fibrosis http://www.irishthoracicsociety.com/images/uploads/ITS\%20Statement $\%$ 20Final.pdf (Last accessed 28 August 2013).

9. Care programs for idiopathic pulmonary fibrosis .Swedish Society for Pulmonary Medicine.

http://www.sImf.se/sites/default/files/VPIL. (Last accessed 28 August $\underline{2013}$

10. Pirfenidone for treating idiopathic pulmonary fibrosis. National Institute for Health and Clinical Excellence. http://guidance.nice.org.uk/TA/Wave23/25/FAD/FinalAppraisalDeterminat ion/pdf/English. (Last accessed 28 August 2013).

11. Potts J, Yogaratnam D. Pirfenidone: a novel agent for the treatment of idiopathic pulmonary fibrosis. Ann Pharmacother 2013; 47: 361-367

* A practical and concise recent review on pirfenidone

12. Schaefer CJ, Ruhrmund DW, Pan L et al. Antifibrotic activities of pirfenidone in animal models. Eur Respir Rev 2011;20:120,85-97 
* Updated review on the antifibrotic effects of pirfenidone in animal models of pulmonary fibrosis.

13. Giri SN, Leonard S, Shi X, al. Effects of pirfenidone on the generation of reactive oxygen species in vitro. J Environ Pathol Toxicol Oncol 1999; 18: 169177

14. Macías-Barragán J, Sandoval-Rodríguez A, Navarro-Partida J et al. The multifaceted role of pirfenidone and its novel targets. Fibrogenesis and Tissue Repair 2010; 3: 16

15. Gurujeyalakshmi G, Hollinger MA, Giri SN. Pirfenidone inhibits PDGF isoforms in bleomycin hamster model of lung fibrosis at the translational level. Am J Physiol Lung Cell Mol Physiol 1999; 276: L311-L318.

16. Nakazato $\mathrm{H}$, Oku H, Yamane $\mathrm{S}$ et al. A novel antifibrotic agent pirfenidone suppresses tumor necrosis factor- $\alpha$ at the translational level. Eur $\mathrm{J}$ Pharmacol 2002; 446: 177-185

17. Oku $H$, Shimizu $T$, Kawabata $T$, et al. Antifibrotic action of pirfenidone and prednisolone: different effects on pulmonary cytokines and growth factors in bleomycininduced murine pulmonary fibrosis. Eur J Pharmacol 2008; 590: 400-408.

18. Oku H, Nakazato $\mathrm{H}$, Horikawa $\mathrm{T}$, et al. Pirfenidone suppresses tumor necrosis factor-a, enhances interleukin-10 and protects mice from endotoxic shock. Eur J Pharmacol 2002; 446: 167-176. 
19. Tian XL, Yao W, Guo ZJ e al. Low dose pirfenidone suppresses transforming growth factor beta-1 and tissue inhibitor of metalloproteinase-1, and protects rats from lung fibrosis induced by bleomycin. Chin Med Sci J 2006; 21: $145-151$

20. Ozes ON, Blatt LM. Development of a high throughput collagen assay using a cellular model of idiopathic pulmonary fibrosis. Chest 2006; $130: 230 \mathrm{~S}$

21. Nayayama S, Mukae $H$, Sakamoto $M$ et al. Pirfenidone inhibits the expression of HSP47 in TGF- $\beta 1$-stimulated human lung fibroblasts. Life Sci 2008; 82: 210-217.

22. Hisatomi K, Mukae H, Sakamoto N. Pirfenidone inhibits TGF- $\beta-1$ induced overexpression of collagen type I and heat shock protein 47 in A549 cells. BCM Pulm Med 2012, 12: 24

23. Jackson RM, Gomez Marin O. Development and utility of pirfenidone in the treatment of idiopathic pulmonary fibrosis: review of preclinical science and recent clinical trials. Trasplant Res Risk Manag 2011; 3:55-63.

24. Shi S, Wu J, Chen $\mathrm{H}$ et al. Single and multiple-dose pharmacokinetics of pirfenidone, an antifibrotic agent, in healthy Chinese volunteers. J Clin Pharmacol 2007; 47: 1268-1276 
25. Huang NY, Ding L, Wang J, et al. Pharmacokinetics, Safety and Tolerability of Pirfenidone and its Major Metabolite after Single and Multiple Oral Doses in Healthy Chinese Subjects under Fed Conditions. Drug Res (Sturg), 2013 Apr 11.

26. Rubino CM, Bhavnani SM, Ambrose PG et al. Effect of food and antiacids on the pharmacokinetics of pirfenidone in older healthy adults. Pulm Pharmacol ther 2009, 22: 279-285

27. InterMune. Esbriet_(pirfenidone): EU summary of product characteristics [online]. Available from URL:

http://www.ema.europa.eu/docs/en_GB/document_library/EPAR_-_Pro duct_Information/human/002154/WC500103049.pdf. (Last accessed 28 August 2013).

28. Taniyama M, Ohbayashi $S$, Narita $M$, et al. Pharmacokinetics of an antifibrotic agent, pirfenidone, in haemodialysis patients. Eur J Clin Pharmacol. $1997 ; 52: 77-8$.

29. Raghu G, Johnson CJ, Lockhart D et al. Treatment of idiopathic pulmonary fibrosis with a new antifibrotic agent, pirfenidone. Am J Respir Crit Care Med 1999; 159: 1061-1069 
30. Azuma A, Nukiwa T, Tsuboi E et al. Double-blind, placebo controlled trial of pirfenidone in patients with idiopathic pulmonary fibrosis. Am J Respir. Crit Care Med 2005; 171: 1040-1047

31. Taniguchi $H$, Ebina $M$, Kondoh $Y$ et al. Pirfenidone in idiopathic pulmonary fibrosis. Eur Respir J 2010; 35: 821-829

${ }^{* *}$ Pivotal phase III clinical trial of pirfenidone in IPF

32. Azuma A, Tanaguchi $\mathrm{Y}$, Ogura $\mathrm{T}$ et al. Exploratory analysis of a phase III trial of pirfenidone identifies a subpopulation of patients with idiopathic pulmonary fibrosis as benefiting from treatment. Respir Res 2011; 12: 143.

33. Taniguchi $\mathrm{H}$, Kondo $\mathrm{H}$, Ebina $\mathrm{M}$ et al. The clinical significance of $5 \%$ change in vital capacity in patients with idiopathic pulmonary fibrosis: extended analysis of the pirfenidone trial. Respir Res 2011; $12: 93$

34. Noble PW, Albera C, Bradford WZ et al. Pirfenidone in patients with idiopathic pulmonary fibrosis (CAPACITY): two randomised trials. Lancet 2011; 377: $1760-1769$

** The most outstanding phase III clinical trials of pirfenidone in IPF

35. Richeldi L, du Bois RM. Pirfenidone in idiopathic pulmonary fibrosis: the CAPACITY program. Expert Rev Respir Med 2011; 5:473-481 
36. Spagnolo P, Del Giovane C, Luppi F et al. Non-steroid agents for idiopathic pulmonary fibrosis. Cochrane Database Syst Rev 2010;9:CD003134

** Excellent review on the effects of non-steroid drugs in IPF

37. Costabel U, Albera C, Bradfor W et al. Analysis of lung function and survival in RECAP: an open-label extension study of pirfenidone in patients with idiopathic pulmonary fibrosis. Eur Respir J 2012; 40, Supl 56: 511s

38. Okuda R, Hagiwara E, Baba T et al. Safety and efficacy of pirfenidone in idiopathic pulmonary fibrosis in clinical practice. Resp. Med 2013; 107: 14311437

39. Bonella F, Wessendorf TE, Costabel U. Clinical experience with pirfenidone for the treatment of idiopathic pulmonary fibrosis. Dtsch Med Wochenschr 2013; 138: $518-523$

40. Iwasawa T, Ogura T, Sakai F et al. CT analysis of the effect of pirfenidone in patients with idiopathic pulmonary fibrosis. Eur J Radiol 2012; Mar 30 (Epub ahead of print).

41. Jiang $\mathrm{CH}$, Huang $\mathrm{H}$, Liu $\mathrm{J}$ et al. Adverse events of pirfenidone for the treatment of pulmonary fibrosis: a meta-analysis of randomized controlled trials. PLoS One 7(10): e47024.

** Updated review on pirfenidone's adverse effects 
42. Gahl WA, Brantly M, Troendle J et al. Effect of pirfenidone on the pulmonary fibrosis of Hermansky-Pudlak syndrome. Mol Genet Metab 2002; 76: 234-242.

43. O'Brien K, Troendle J, Gochuico BR et al. Pirfenidone for the treatment of Hermansky-Pudlak syndrome pulmonary fibrosis. Mol Genet Metab 2011: 103: 128-134.

44. InPharm. First idiopathic pulmonary fibrosis drug approved (on line). Available from URL: http://www.inpharm.com/news/149931/intermuneidiopathic-pulmonary-fibrosis-esbriet-pirfenidone (Last accesed 28 August 2013). 
Table 1.- Pirfenidone. Most commonly reported adverse reactions (> 10\% patients)*

\begin{tabular}{|l|c|c|}
\hline \multicolumn{1}{|c|}{ Adverse event } & Pirfenidone $2403 \mathrm{mg} /$ day & Placebo \\
\hline Nausea & $32.8^{* *}$ & 13.3 \\
\hline Rash & 28.7 & 8.6 \\
\hline Fatigue & 22.3 & 13.3 \\
\hline Diarrhea & 21.7 & 13.5 \\
\hline Dyspepsia & 16.8 & 5.5 \\
\hline Photosensitivity & 12.2 & 1.7 \\
\hline
\end{tabular}

* Data from clinical studies including 1345 healthy volunteers and patients (27).

** $\%$ of patients 
1

2

Table 2.- Potential drug interactions of pirfenidone

CYP1A2 inhibitors

Fluvoxamine

Ciprofloxacin

Propafenone

Grapefruit juice

CYP2C9 inhibitors

Amiodarone

Fluconazole

Voriconazole

CYP2C19 inhibitors

Chloramphenicol

CYP2D6 inhibitors

Fluoxetine

Paroxetine

Quinidine

CYP1A2 inducers

Tobacco smoke

Omeprazole

\begin{abstract}
Abbreviations: CYP, Cytochrome
\end{abstract}




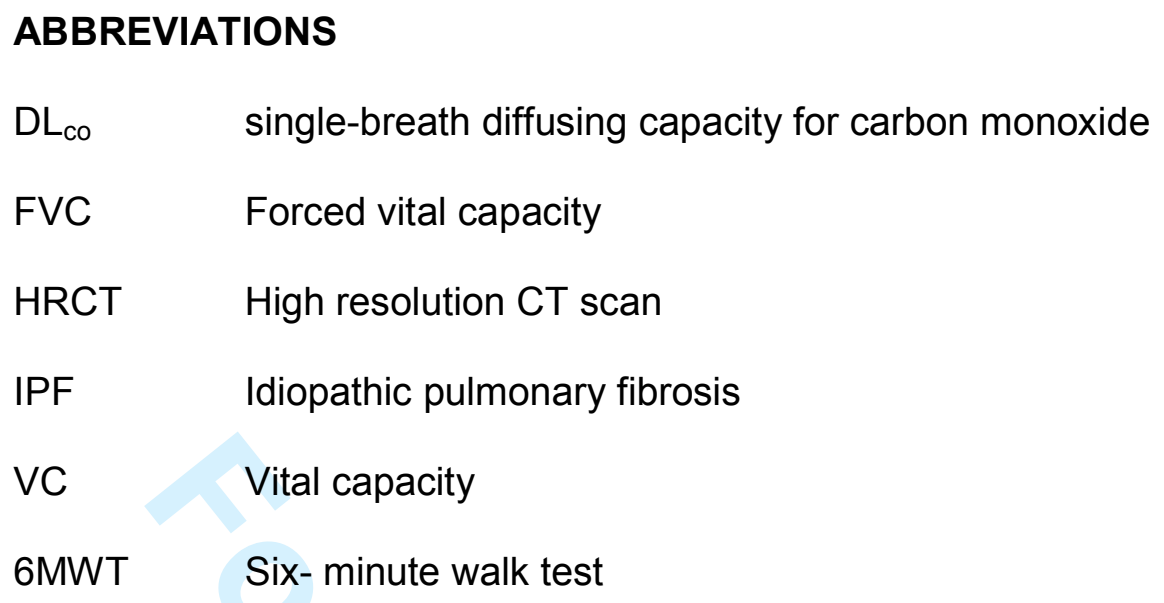


Table 1.- Pirfenidone. Most commonly reported adverse reactions (> $10 \%$ patients)*

\begin{tabular}{|l|c|c|}
\hline \multicolumn{1}{|c|}{ Adverse event } & Pirfenidone 2403 mg/ day & Placebo \\
\hline Nausea & $32.8^{* *}$ & 13.3 \\
\hline Rash & 28.7 & 8.6 \\
\hline Fatigue & 22.3 & 13.3 \\
\hline Diarrhea & 21.7 & 13.5 \\
\hline Dyspepsia & 16.8 & 5.5 \\
\hline Photosensitivity & 12.2 & 1.7 \\
\hline
\end{tabular}

* Data from clinical studies including 1345 healthy volunteers and patients (27).

** $\%$ of patients

URL: http://mc.manuscriptcentral.com/eoop Email: Amina.AbdelLatif@informa.com 
Table 2.- Potential drug interactions of pirfenidone

CYP1A2 inhibitors

Fluvoxamine

Ciprofloxacin

Propafenone

Grapefruit juice

CYP2C9 inhibitors

Amiodarone

Fluconazole

Voriconazole

CYP2C19 inhibitors

Chloramphenicol

CYP2D6 inhibitors

Fluoxetine

Paroxetine

Quinidine

CYP1A2 inducers

Tobacco smoke

Omeprazole

Abbreviations: CYP, Cytochrome

URL: http://mc.manuscriptcentral.com/eoop Email: Amina.AbdelLatif@informa.com 


\section{Declaration of interest:}

Author 1 declares that he has received funding for given lectures at educational events and/or for scientific advice and / or research from Intermune, Actelion and Boehringer Ingelheim in relation to the subject of the manuscript.

Author 2: Intermune.

Author 3: Intermune, Zambon, Boehringer Ingelheim. 


\section{PIRFENIDONE FOR THE TREATMENT OF IDIOPATHIC PULMONARY FIBROSIS}


Antoni Xaubet, MD, PhD (corresponding author)

Servei de Pneumologia. Hospital Clínic. Barcelona. Universitat de Barcelona Centro de Investigaciones Médicas en Red de Enfermedades Respiratorias (CIBERES)

Servei de Pneumologia. Hospital Clinic. Villarroel 170. Barcelona 08036. Spain

Phone 60044756

FAX 34932275455

e-mail: axaubet@clinic.ub.es

Anna Serrano Mollar, PhD

Institut d'Investigacions Biomèdiques de Barcelona. Consejo Superior de Investgaciones Científicas. Barcelona. Centro de Investigaciones Médicas en Red de Enfermedades Respiratorias (CIBERES).

Julio Ancochea, MD, PhD

Servicio de Neumología. Hospital Universitario de La Princesa. Madrid 


\begin{abstract}
Introduction. Idiopathic pulmonary fibrosis (IPF) is a chronic, progressive, and lethal fibrosing interstitial pneumonia. The median survival from the onset of the symptoms is $2.8-4.2$ years and the 5 -year survival rate is $20 \%$. Its poor prognosis, combined with the scarcity of treatment options, provides a strong rationale for the development of novel therapeutic strategies. During the last decade there has been a huge rise in clinical trials with antifibrotic drugs, although only pirfenidone (Esbriet) has shown a beneficial effect.
\end{abstract}

Areas covered. This article reviews the medical literature on the effectiveness and safety of pirfenidone in IPF, by means of a PubMed search from 1995 to present, completed by some data on file from the manufacturer.

Expert opinion. Pirfenidone is the only antifibrotic drug approved for the treatment of IPF. Pirfenidone presents a meaningful clinical effect on reductions in the decrease in forced vital capacity (FVC), six minute walk test (6MWT) distance and mortality, and it improves the progression-free survival in IPF patients with mild-moderate disease. Pirfenidone is well tolerated, with the most common side-effects being gastrointestinal discomfort and photosensitivity. Pirfenidone has a favorable benefit-risk profile and represents a suitable treatment option for patients with mild-moderate IPF.

Key words. Antifibrotic drugs, Idiopathic Pulmonary Fibrosis, Pirfenidone 


\section{INTRODUCTION}

IPF is a chronic, progressive, and lethal fibrosing interstitial pneumonia, limited to the lung and associated with the histopathological and/or radiological pattern of usual interstitial pneumonia (1). Although its etiology is unknown and the pathogenesis only partly understood, our current knowledge suggests that damage to the alveolar epithelium is probably an important early event followed by an aberrant healing response. The median age at diagnosis is 66 years, with a male predominance, and prevalence has been increasing in recent years. The incidence of IPF is estimated to be between 4.6 and 7.4 cases $/ 100,000$, and the prevalence is between 13 cases/100,000 for females and 20 cases/100,000 for males (1). IPF is characterized by a progressive decline in pulmonary function, which quickly leads to respiratory failure and death, although some patients may have episodes of acute respiratory worsening despite previous stability. With a median survival from the onset of the symptoms of 2.8-4.2 years and a 5-year survival rate approaching $20 \%$, IPF is more lethal than many malignant diseases. The poor prognosis, combined with the scarcity of treatment options, provides a strong rationale for the development of novel therapeutic strategies for this disease. During the last decade there has been a huge rise in clinical trials with antifibrotic drugs. In phase III randomized clinical trials with interferon gamma 1-b, pirfenidone, macitentan, bosentan, ambrisentan, warfarin, triple therapy (n-acetylcisteine, glucocorticoids, azathitoprine), etanercept and sildenafil, only pirfenidone (Esbriet) has shown any beneficial effects in patients with IPF (2-4). Several national guidelines on IPF diagnosis and treatment from Spain, Germany, Denmark, Sweden, Austria, and Ireland, have recently recommended pirfenidone as first-choice therapeutic agent in IPF patients with 
mild-moderate disease, defined as FVC predicted $>50 \%(5-9)$. In some other European countries, such as Italy, pirfenidone is however, already commercially available. The National Institute for Health and Clinical Excellence (NICE) recommends pirfenidone as a therapeutic option in IPF patients with FVC predicted between 50 and $80 \%$ (10).

\section{OVERVIEW OF THE MARKET}

Pirfenidone is the only pharmacological agent approved for the treatment of mild-moderate IPF (11). Nowadays, there is no drug available for the treatment of patients with IPF in an advance stage.

\section{INTRODUCTION TO PIRFENIDONE}

Studies performed in animal models of pulmonary fibrosis and in human pulmonary cell-based assays have demonstrated that pirfenidone is a pleiotropic molecule with antifibrotic, anti-inflammatory and antioxidant effects (12). The antioxidant effect occurs through its ability to scavenge reactive oxygen species in vitro (13). Studies in animal models of pulmonary fibrosis have shown that pirfenidone attenuates a range of inflammatory and profibrotic molecules while down-regulating histological markers of fibrosis and cellular proliferation (14). Pirfenidone inhibits the expression of transforming growth factor (TGF)- $\beta$ and the tissue inhibitor of metalloproteinase-1 (TIMP-1), and blocks the proliferative effects of platelet-derived growth factor (PDGF). Furthermore, pirfenidone inhibits the release of proinflammatory cytokines such as, tumor necrosis factor (TNF)- $\alpha$, interleukin (IL-1)- $\beta$, IL-6, IL-8 and IL-12, as well as increasing the expression of the anti-inflammatory cytokine IL-10, 
attenuating the release of chemotactic cytokines and reducing the accumulation of inflammatory cells in response to different stimuli (14-19).

In addition to the modulation of mediators in animal models, cell-based assays have demonstrated that pirfenidone inhibits the expression of heat shock protein (HSP) 47 in cultured normal lung fibroblasts stimulated with TGF- $\beta-1$; it was also able to inhibit the expression of collagen in fibroblasts isolated from IPF patients $(20,21)$. Moreover, pirfenidone inhibits the overexpression of collagen type I and HSP47 in the alveolar epithelial cell line (A549 cells) (22).

\subsection{CHEMISTRY}

Pirfenidone is an orally bioavailable synthetic molecule. Pirfenidone is a heterocyclic pyridine (5-methyl-1-phenyl-2-[1H]-pyridine) with a molecular weight of 185.22. Pyridines are derived from coal combustion, and can be synthesized from aldehyde and ammonia. They are highly soluble in dimethylsulfoxide, twice in water and chloroform, and in alcohol. In aqueous solution the maximum possible concentration is $2 \%(23)$.

\subsection{PHARMACOKINETICS AND METABOLISM}

Oral pirfenidone has linear pharmacokinetics over the dose range of 200-600 mg. (24). After a single dose of pirfenidone with food at the recommended maintenance dose of $801 \mathrm{mg}$, the mean maximum plasma concentration $\left(\mathrm{C}_{\max }\right)$ is $7.9 \mathrm{mg} / \mathrm{L}$ in healthy adults. Absorption occurs quickly, the time to maximum $\left(T_{\max }\right)$ values being achieved in $30-60 \min (25)$. Median time to $C_{\max }$ is $3.5 \mathrm{~h}$. However, the $\mathrm{C}_{\max }$ is significantly lower when the drug is administered in the fed versus fasting states (26). Concomitant intake of food reduce by $20 \%$ the rate 
and extent of absorption, which is associated with a better tolerability to pirfenidone tt has been shown that healthy volunteers who received pirfenidone with food had and a lower incidence of gastrointestinal adverse events, than those who received pirfenidone without food. (24). In clinical trials, at concentrations of $1-1000 \mathrm{mg} / \mathrm{L}, 50-58 \%$ of pirfenidone bound to plasma proteins, predominantly albumin (27). Pirfenidone is predominantly metabolized by the cytochrome (CYP) P450 CYP1A2 enzyme, although other CYP enzymes, including CYP2C9, 2C19, 2D6, and 2E, also contribute to the metabolism. The major metabolite of pirfenidone is 5-carboxy-pirfenidone which is inactive. After a single $801 \mathrm{mg}$ dose, the terminal elimination half-life was 2.9 h (without food) and $2.4 \mathrm{~h}$ (with food). No significant gender differences were noted for the pharmacokinetic variables (26). Eighty percent of the administered dose was excreted in the urine primarily as 5-carboxy-pirfenidone and less than $1 \%$ of the dose recovered in the urine was unaltered, after $6 \mathrm{~h}$ of administration. Pirfenidone bioavailability is increased by a mean of $60 \%$ in patients with moderate hepatic function impairment. No clinical relevant changes have been observed in pharmacokinetics in patients with mild to severe renal function impairment. However, the drug is contraindicated in patients with severe renal impairment (creatinine clearance of $<30 \mathrm{~mL} / \mathrm{min}$ ) or end stage renal disease requiring dialysis (28).

Pirfenidone may interact with drugs that inhibit CYP1A2, particularly fluvoxamine, ciprofloxacin, and propafenone. Consumption of grapefruit also inhibits CYP1A2. Concomitant treatment with inhibitors of other CYP isoenzymes involved in the metabolism of pirfenidone (fluconazole, chloramfenicol, fluoxetine, paroxetine, amiodarone) may interact with 
pirfenidone. Pirfenidone can also interact with CYP1A2, inducers, such as tobacco smoke and omeprazole (27) (Table 1).

\section{CLINICAL EFFICACY}

\subsection{Clinical trials}

In a prospective, open-label phase II study, pirfenidone was evaluated for its tolerability and usefulness in patients with advanced IPF, progressive disease and lack of response to conventional therapy (prednisone with or without immunosuppressives). Fifty-four patients were followed for mortality, changes in lung function and adverse effects. Patients whose lung function had deteriorated before enrolment appeared to stabilize after beginning the treatment. Patients with higher single-breath diffusing capacity for carbon monoxide $\left(\mathrm{DL}_{\mathrm{co}}\right)$ (> $30 \%$ predicted) at entry had longer survival rates. The adverse effects were minor (29).

The first large-scale trial of pirfenidone was a Japanese multicenter randomized placebo-controlled phase II study of 107 subjects who received either pirfenidone $600 \mathrm{mg}$ three times daily $(n=72)$ or placebo $(n=35)$. The trial was designed to run for 1 year. Although there was a significant decrease in the decline of FVC in the pirfenidone group, 9-month interim results showed that five subjects from the placebo group had suffered acute exacerbation of the disease compared to none in the pirfenidone group, and the study was halted (30).

A phase III clinical trial conducted over 52 weeks was also developed in Japan. A total of 275 patients were randomized to either high-dose (1800 mg/day) or low-dose (1200 mg/day) pirfenidone or placebo at the ratio of 2:1:2. Significant 
differences were observed in vital capacity (VC) decline (primary end-point) between the placebo group and the high-dose group and improved progressionfree survival time was seen in the high-dose group. (31). In order to find out which patients specifically benefit from pirfenidone, an additional exploratory analysis was performed with the data of this trial. Significant efficacy of pirfenidone in reducing the decline in VC was seen in a subpopulation with $\% \mathrm{VC} \geq 70 \%$ and oxygen saturation at baseline $\left(\mathrm{SpO}_{2}\right)<90 \%$. These are mutually conflicting characteristics. According to the author's opinion, the desaturation exhibited by the subgroup with the better-preserved lung function may have been due to the development of fibrosis with inflammatory edema and not to established fibrosis. In this subpopulation, pirfenidone also suppressed cough and dyspnea (32). Recently, marginal decline in FVC (5\%) has been reported as being associated with poor outcome in IPF. In the same clinical trial, Taniguchi et al. sought to evaluate the efficacy of pirfenidone from the aspects of $5 \%$ changes in VC. When $5 \%$ change in VC was used as an index instead of the $10 \%$ change, the efficacy of pirfenidone could be evaluated with higher sensitivity over the 12 months study (33).

The CAPACITY phase III trials consisted of two concurrent multinational randomized double-blind placebo-controlled phase III trials (004 and 006) conducted over 72 weeks and designed to evaluate the safety and efficacy of pirfenidone in patients with mild-moderate disease $(34,35)$. In trial 004, 174 patients were assigned to high-dose pirfenidone (2403 mg/day), 87 to low dose (1197 mg/day) and 174 to placebo. In study 006, 171 patients were assigned to high-dose pirfenidone (2403 mg/day), and 173 to placebo. In study 004, the higher dose of pirfenidone met the primary endpoint, decreasing the decline in 
FVC. In contrast, trial 006 failed to show a significant reduction in FVC, although there was a reduced decline in distance walked in the 6MWT (secondary endpoint). The differences observed between the two studies in the effect of pirfenidone may be explained by the fact that the subjects in the 006 placebo group had a slower rate of decline compared to those in 004 . When 004 and 006 study data were pooled, pirfenidone showed meaningful clinical effects on FVC \% predicted (decrease in the decline by $30 \%$ ), progression free survival and 6MWT distance at the dose of $2403 \mathrm{mg} / \mathrm{day}$. Although these trials were not empowered to detect an effect on mortality, IPF-related mortality was reduced by $28 \%$. A Cochrane review encompassing the two Japanese trials and the CAPACITY studies, has demonstrated that pirfenidone improves progressionfree survival by $30 \%$. (36). However, there are still no data for having longer overall survival in patients treated with pirfenidone.

The RECAP study is an ongoing open-label extension study evaluating the long-term administration of pirfenidone in patients who completed phase III CAPACITY program. Preliminary results in 178 patients show that FVC and survival outcomes in patients newly treated with pirfenidone in the RECAP study were similar to those treated in the CAPACITY trials. These data provide further evidence to support the beneficial effect of pirfenidone in IPF (37). A phase III study comparing pirfenidone with placebo in patients with mild-tomoderate IPF is currently underway in the US (ASCEND trial, NCT01366299). The PANORAMA study (A Randomized, Double-Blind, Placebo-Controlled, Phase II Study of the Safety and Tolerability of $N$-Acetylcysteine in Patients with Idiopathic Pulmonary Fibrosis with Background Treatment of Pirfenidone, EUDRACT 2012-000564-14) is also ongoing. The objective of this trial is to 
assess the safety and tolerability of the treatment with $\mathrm{N}$-Acetylcysteine in patients with mild-to-moderate IPF on background treatment of pirfenidone.

\subsection{Posology and method of administration}

Pirfenidone is indicated in adults for the treatment of mild-to-moderate IPF (FVC $>50 \%$ predicted (5-9). The National Institute for Health and Clinical Excellence recommends pirfenidone in patients with FVC between $50 \%$ and $80 \%$ predicted (10). The approved dose is $2403 \mathrm{mg} /$ day. Each capsule contains $267 \mathrm{mg}$. Upon initiating treatment, the dose should be titrated to the recommended daily dose over a 14-day period: days 1 to 7 , one capsule three times a day (801 mg/day); days 8 to 14, two capsules, three times a day (1602 mg/day) and day 15 onward, three capsules, three times a day (2403 mg/day). The drug should be taken with food to reduce the possibility of gastrointestinal adverse events and dizziness (27). The recommended treatment duration is at least 12 months. If there is improvement or stabilization of the disease, the treatment should be continued. In case of deterioration, the advisability of continuing treatment or instigating other therapeutic strategies should be considered for each patient. However, it It is recommended that treatment should be discontinued if there is evidence of disease progression, that is, a decline in percentage predicted FVC of $10 \%$ or more within any 12 month period $(5,10)$. However, the decision of discontinuation should be considered according to the individual benefit and adverse effects under the comprehensive evaluation. 


\subsection{Post marketing studies}

Open-label studies have been developed, to confirm the efficacy, safety and tolerability of pirfenidone and to optimize its indications.

A European Named Patient Program (NPP) was established by Intermune to make pirfenidone accessible to patients with IPF during the period between the authorization of the drug and its availability upon prescription. The results of this program have not yet been published. The PASSPORT study (PostAuthorisation Safety Study of Esbriet(i) (Pirfenidone): A Prospective Observational Registry to Evaluate Long-Term Safety in a Real-World Setting) is also ongoing; its objective is to evaluate the long-term safety of pirfenidone. In a retrospective study, Okuda et al. (38) have described the effects of pirfenidone, at the dose of $1800 \mathrm{mg} /$ day during 6 months, in 76 patients with mild-to-severe IPF. Pirfenidone tended to attenuate the degree of decline in FVC, particularly in the subgroup of patients with FVC $<60 \%$ and showed greater efficacy in patients with a decline in FVC $>150 \mathrm{ml}$ during the 6-months period before the start of therapy. Thus, the degree of disease progression prior to the initiation of therapy had an impact on the response to pirfenidone. The most frequent adverse effects were anorexia (42\%), increase in gamma glutamyl transpeptidase (22\%), fatigue (14\%) and photosensitivity (18\%). All the reported adverse effects were, however, mild, reversible and left no sequelae. Moreover, Bonella et al. (39), have reported data on the safety and efficacy of pirfenidone $(2403 \mathrm{mg} / \mathrm{day}$ ) in 45 patients with mild-moderate IPF (mean duration of treatment 48 weeks). Sixteen patients (35\%) received pirfenidone as monotherapy and $29(65 \%)$ in combination with corticosteroids and /or $\mathrm{N}$ acetylcisteine. The course of the disease was stable during treatment with 
pirfenidone in two out of every three patients. Twenty-six patients (58\%) suffered from side effects, mostly gastrointestinal, but pirfenidone was discontinued because of side effects in only six patients. The results of this study confirm the efficacy and safety of pirfenidone in the treatment of IPF. In another study, Inoue et al. have reported the efficacy and safety profile of pirfenidone in more than 1,300 Japanese patients with IPF. Pirfenidone kept the decline in VC to a minimum (mean change $-0.07 \mathrm{~L}$ ) in patients treated 6 months o longer. Incidences of decreased appetite, photosensitivity reaction and nausea were $29 \%, 15 \%$ and $8.3 \%$ respectively, although most of these adverse drug reactions were manageable (40).

Iwasawa et al. (41) investigated the usefulness of high resolution CT scan (HRCT) in the imaging assessment of the response to pirfenidone therapy. Seventy-eight patients (38 treated with pirfenidone and 40 matched controls) were given HRCT on two occasions, with a one-year interval in between. A significantly larger proportion of patients treated with pirfenidone showed more stable pulmonary function parameters than the controls (65.6\% vs $37.5 \%)$. The decline in VC correlated with the increase in fibrotic lesions in CT scan. These results suggest that CT scan may be useful for evaluating the pirfenidoneinduced slowing progression of pulmonary fibrosis.

\section{SAFETY AND TOLERABILITY}

Jiang et al (42) have reported the results of a meta-analysis to analyze the safety profile of pirfenidone for treating pulmonary fibrosis. Six-randomized controlled trials were analyzed. A total of 1073 patients were enrolled, 561 in the pirfenidone group, and 512 in the placebo group. Four trials assessed the 
treatment of IPF and the remaining two studies, assessed the treatment of Hermansky-Pudlak syndrome $(30,31,34,43,44)$. The combined results of the six trials revealed that the pirfenidone group had a significantly higher rate of gastrointestinal, neurological and dermatological adverse events. However, adverse effects were generally mild or moderate in severity and without any clinically significant consequences. Most of the adverse effects disappeared with a decrease in the dose or temporary discontinuation of medication.

The most commonly reported $(\geq 10 \%)$ adverse reactions during treatment with pirfenidone at a dose of $2403 \mathrm{mg} /$ day are nausea, rash, fatigue, diarrhea, dyspepsia and photosensitivity reaction (table 2). Less frequent adverse effects are anorexia, insomnia, hot flush and alteration in the hepatic enzymes alanin and aspartate aminotransferases, and gamma glutamyl transferase (27).

Gastrointestinal events are less frequent if the drug is administered with food. Patients with photosensitivity reaction or rash should use protective sun creams, wear clothing that protects against sun exposure and avoid direct sunlight. In case of adverse events, the dose of pirfenidone should be adjusted or the treatment discontinued, according to the established recommendations. There are no data on the use of pirfenidone during pregnancy (27).

Pirfenidone is contraindicated in case of hypersensitivity to the drug or to any of its excipients, concomitant use of fluvoxamine, severe hepatic impairment or end-stage liver disease or severe renal impairment or end-stage renal disease requiring dialysis. Pirfenidone should be used with caution in patients treated with inhibitors or inducers of CYP1A2 (table 1) (27). 


\section{REGULATORY AFFAIRS}

Pifenidone is an orally administered pyridine that was granted orphan drug approval in the European Union for the treatment of mild-to-moderate IPF in 2011 and it is the only pharmacological agent available for this indication (45). In Europe, it is marketed under the brand name Esbriet ${ }^{\circledR}$ by Intermune Inc. In Japan, pirfenidone has been approved for marketing since 2008 under the brand name Pirespa ${ }^{\circledR}$ by Shionogi \& Co. In 2010, the Food and Drug Administration (FDA) denied approval for pirfenidone. A new drug application to the FDA is expected depending on the results of the ongoing phase III trial ASCEND (Efficacy and safety of Pirfenidone in Patients with Idiopathic Pulmonary Fibrosis).

\section{CONCLUSION}

IPF is a chronic, progressive, and lethal fibrosing interstitial pneumonia. Its poor prognosis, combined with the scarcity of treatment options, provides a strong rationale for the development of novel therapeutic strategies for this disease. During the last decade several clinical trials with antifibrotic drugs have been developed, although only pirfenidone has shown a beneficial effect. Recent national guidelines and the National Institute for Health and Clinical Excellence recommend pirfenidone as first-choice therapeutic agent in IPF patients with mild-moderate disease, defined as FVC $>50 \%$ of predicted. Pirfenidone's adverse-effect profile is acceptable in a disease as severe as IPF. Nowadays, pirfenidone is the only pharmacological agent approved for the treatment of mild-moderate IPF. 


\section{EXPERT OPINION}

IPF is a rare and orphan disease characterized by a progressive lung fibrosis with a median survival from the onset of the symptoms of 2.8 to 4.2 years. An international consensus statement for the diagnosis and treatment of IPF was published in 2011. In this consensus, the diagnostic criteria of IPF were redefined and new decisions for diagnosis and clinical management were established. However, the committee did not find sufficient evidence to support the use of any specific pharmacologic therapy for patients with IPF. Since the publication of the 2011 international consensus we have known the final results of several clinical trials with antifibrotic drugs, although only pirfenidone has shown any beneficial effect. Four key clinical trials have endorsed the efficacy and tolerability of pirfenidone. Pirfenidone has shown clinically meaningful effects on decreases in the decline of \% FVC, 6MWT distance and IPF- related mortality, as well as on improvements in progression-free survival. In addition, pirfenidone could prevent symptoms related to disease progression, decrease dependency on oxygen, and reduce hospitalizations. Moreover, post-marketing studies have confirmed these beneficial effects of pirfenidone. Thus, pirfenidone seems to have a modest but measurable effect on slowing the progression of the disease, but there is uncertainty in whether this benefit will persists over time. Therefore, the main goal of long term treatment with pirfenidone is to increase the survival. It is an important point because there are still no data for having longer overall survival of patients treated with pirfenidone. In this context, open-label and placebo-controlled studies are underway to confirm the efficacy and safety of pirfenidone. Pirfenidone is well tolerated, with the most common side-effects being gastrointestinal discomfort and photosensitivity. It is 
considered that the pirfenidone's adverse-effect profile is acceptable in a disease as severe as IPF.

Pirfenidone is the only pharmacological agent approved for the treatment of mild-moderate IPF. Recent national guidelines and the National Institute for Health and Clinical Excellence recommend pirfenidone as first-choice therapeutic agent in IPF patients with mild-moderate disease, defined as FVC > $50 \%$ of predicted. Depending on the results of the ongoing clinical trials, pirfenidone will likely become in the next few years in the main treatment for mild- moderate IPF.

Nowadays, there is no drug available for the treatment of patients with IPF with advanced disease, which is defined by FVC lower than $50 \%$ predicted. To date, the majority of clinical trials in IPF have included only patients with mildmoderate disease. One of the research projects that should be done, is a clinical trial of pirfenidone in IPF patients with advanced disease, with the aim of evaluating its efficacy in this group of patients.

At present, clinical trials are underway with other antifibrotic mediators in patients with IPF, but we still do not know the results. In conclusion, pirfenidone has a favourable benefit-risk profile and represents a suitable treatment option for IPF. 


\section{DRUG SUMMARY BOX}

Drug name

Phase

Indication

Pharmacology description

Route of administration

Chemical structure

Pivotal trials
Pirfenidone

Marketed

mild-moderate idiopathic pulmonary fibrosis antifibrotic, anti-inflammatory, antioxidant

oral<smiles>Cc1ccc(=O)n(-c2ccccc2)c1</smiles> 


\section{REFERENCES}

1. Raghu G, Collard HR, Egan JJ et al. An official ATS/ERS/ALAT statement: idiopathic pulmonary fibrosis: evidence-based guidelines for diagnosis and management. Am J Respir Crit Care Med 2011; 183: 788824.

2. Luppi $F$, Spagnolo $P$, Cerri $S$ et al. The big clinical trials in idiopathic pulmonary fibrosis. Curr Opin Pulm Med 2012; 18: 428-432.

3. Raghu G, Behr J, Brown KK et al. Treatment of idiopathic pulmonary fibrosis with ambrisentan: a parallel randomised trial. Ann Intern Med 2013; 158: 641-649.

4. Raghu G, Million-Rousseau R, Morganti A et al. Macitentan for the treatment of IPF: the randomized controlled MUSIC trial. Eur Respir J 2013; May 16 (Epub ahead of print).

5. Xaubet A, Ancochea J, Bollo E et al. Guidelines for the diagnosis and treatment of idiopathic pulmonary fibrosis. Arch Bronconeumol 2013; 49: 343-353.

6. Behr J, Günther A, Ammenwerth $W$ et al. German Guideline for Diagnosis and Management of idiopathic Pulmonary Fibrosis. Pneumologie 2013; 67:81-111. 
7. Idiopathic pulmonary fibrosis guidelines. Danish Society of Respiratory Medicine www.lungemedicin.dk (Last accessed 29 August 2013).

8. Position Statement from the Irish Thoracic Society on the treatment of idiopathic pulmonary fibrosis: http://www.irishthoracicsociety.com/images/uploads/ITS\%20Statement\% 20Final.pdf (Last accessed 28 August 2013).

9. Care programs for idiopathic pulmonary fibrosis. Swedish Society for Pulmonary Medicine: http://www.slmf.se/sites/default/files/VPIL. (Last accessed 28 August 2013

10.Pirfenidone for treating idiopathic pulmonary fibrosis. National Institute for Health and Clinical Excellence: http://guidance.nice.org.uk/TA/Wave23/25/FAD/FinalAppraisalDeterminat ion/pdf/English. (Last accessed 28 August 2013).

11.Potts J, Yogaratnam D. Pirfenidone: a novel agent for the treatment of idiopathic pulmonary fibrosis. Ann Pharmacother 2013; 47: 361-367.

${ }^{*}$ A practical and concise recent review on pirfenidone

12. Schaefer CJ, Ruhrmund DW, Pan L et al. Antifibrotic activities of pirfenidone in animal models. Eur Respir Rev 2011;20:120,85-97. 
*Updated review on the antifibrotic effects of pirfenidone in animal models of pulmonary fibrosis.

13. Giri SN, Leonard S, Shi X, al. Effects of pirfenidone on the generation of reactive oxygen species in vitro. J Environ Pathol Toxicol Oncol 1999; 18: $169-177$.

14. Macías-Barragán J, Sandoval-Rodríguez A, Navarro-Partida J et al. The multifaceted role of pirfenidone and its novel targets. Fibrogenesis and Tissue Repair 2010; 3: 16.

15.Gurujeyalakshmi G, Hollinger MA, Giri SN. Pirfenidone inhibits PDGF isoforms in bleomycin hamster model of lung fibrosis at the translational level. Am J Physiol Lung Cell Mol Physiol 1999; 276: L311-L318.

16. Nakazato $\mathrm{H}$, Oku $\mathrm{H}$, Yamane $\mathrm{S}$ et al. A novel antifibrotic agent pirfenidone suppresses tumor necrosis factor- $\alpha$ at the translational level. Eur J Pharmacol 2002; 446: 177-185.

17. Oku H, Shimizu T, Kawabata $T$, et al. Antifibrotic action of pirfenidone and prednisolone: different effects on pulmonary cytokines and growth factors in bleomycin-induced murine pulmonary fibrosis. Eur J Pharmacol 2008; 590: 400-408. 
18. Oku $H$, Nakazato $H$, Horikawa $T$, et al. Pirfenidone suppresses tumor necrosis factor-a, enhances interleukin-10 and protects mice from endotoxic shock. Eur J Pharmacol 2002; 446: 167-176.

19. Tian XL, Yao W, Guo ZJ e al. Low dose pirfenidone suppresses transforming growth factor beta-1 and tissue inhibitor of metalloproteinase-1, and protects rats from lung fibrosis induced by bleomycin. Chin Med Sci J 2006; 21: 145-151.

20. Ozes ON, Blatt LM. Development of a high throughput collagen assay using a cellular model of idiopathic pulmonary fibrosis. Chest 2006; 130 : $230 S$.

21. Nayayama S, Mukae $H$, Sakamoto $M$ et al. Pirfenidone inhibits the expression of HSP47 in TGF- $\beta 1$-stimulated human lung fibroblasts. Life Sci 2008; 82: 210-217.

22. Hisatomi K, Mukae H, Sakamoto N. Pirfenidone inhibits TGF- $\beta-1$ induced overexpression of collagen type I and heat shock protein 47 in A549 cells. BCM Pulm Med 2012, 12: 24.

23. Jackson RM, Gomez Marin O. Development and utility of pirfenidone in the treatment of idiopathic pulmonary fibrosis: review of preclinical science and recent clinical trials. Trasplant Res Risk Manag 2011; 3:5563. 
24. Shi S, Wu J, Chen $\mathrm{H}$ et al. Single and multiple-dose pharmacokinetics of pirfenidone, an antifibrotic agent, in healthy Chinese volunteers. J Clin Pharmacol 2007; 47: 1268-1276.

25. Huang NY, Ding L, Wang J, et al. Pharmacokinetics, Safety and Tolerability of Pirfenidone and its Major Metabolite after Single and Multiple Oral Doses in Healthy Chinese Subjects under Fed Conditions. Drug Res (Sturg), 2013 Apr 11.

26. Rubino CM, Bhavnani SM, Ambrose PG et al. Effect of food and antiacids on the pharmacokinetics of pirfenidone in older healthy adults. Pulm Pharmacol ther 2009, 22: 279-285.

27.InterMune. Esbriet_(pirfenidone): EU summary of product characteristics [online]. $\quad$ Available $\quad$ from $\quad$ URL: http://www.ema.europa.eu/docs/en GB/document library/EPAR - Pro duct_Information/human/002154/WC500103049.pdf. (Last accessed 28 August 2013).

28. Taniyama M, Ohbayashi S, Narita M, et al. Pharmacokinetics of an antifibrotic agent, pirfenidone, in haemodialysis patients. Eur $\mathrm{J}$ Clin Pharmacol. 1997; 52:77-8. 
29. Raghu G, Johnson CJ, Lockhart D et al. Treatment of idiopathic pulmonary fibrosis with a new antifibrotic agent, pirfenidone. Am J Respir Crit Care Med 1999; 159: 1061-1069.

30.Azuma A, Nukiwa T, Tsuboi E et al. Double-blind, placebo controlled trial of pirfenidone in patients with idiopathic pulmonary fibrosis. Am J Respir. Crit Care Med 2005; 171: 1040-1047.

31.Taniguchi $H$, Ebina $M$, Kondoh $Y$ et al. Pirfenidone in idiopathic pulmonary fibrosis. Eur Respir J 2010; 35: 821-829.

** Pivotal phase III clinical trial of pirfenidone in IPF.

32. Azuma A, Tanaguchi Y, Ogura T et al. Exploratory analysis of a phase III trial of pirfenidone identifies a subpopulation of patients with idiopathic pulmonary fibrosis as benefiting from treatment. Respir Res 2011; 12 : 143.

33. Taniguchi $\mathrm{H}$, Kondo $\mathrm{H}$, Ebina $\mathrm{M}$ et al. The clinical significance of $5 \%$ change in vital capacity in patients with idiopathic pulmonary fibrosis: extended analysis of the pirfenidone trial. Respir Res 2011; 12: 93.

34. Noble PW, Albera C, Bradford WZ et al. Pirfenidone in patients with idiopathic pulmonary fibrosis (CAPACITY): two randomised trials. Lancet 2011; 377: 1760-1769.

** The most outstanding phase III clinical trials of pirfenidone in IPF. 
35. Richeldi L, du Bois RM. Pirfenidone in idiopathic pulmonary fibrosis: the CAPACITY program. Expert Rev Respir Med 2011; 5:473-481.

36. Spagnolo P, Del Giovane C, Luppi F et al. Non-steroid agents for idiopathic pulmonary fibrosis. Cochrane Database Syst Rev 2010;9:CD003134.

${ }^{* *}$ Excellent review on the effects of non-steroid drugs in IPF.

37. Costabel U, Albera C, Bradfor $\mathrm{W}$ et al. Analysis of lung function and survival in RECAP: an open-label extension study of pirfenidone in patients with idiopathic pulmonary fibrosis. Eur Respir J 2012; 40, Supl 56: $511 \mathrm{~s}$.

38. Okuda R, Hagiwara E, Baba T et al. Safety and efficacy of pirfenidone in idiopathic pulmonary fibrosis in clinical practice. Resp. Med 2013; 107: $1431-1437$.

39.Bonella F, Wessendorf TE, Costabel U. Clinical experience with pirfenidone for the treatment of idiopathic pulmonary fibrosis. Dtsch Med Wochenschr 2013; 138: 518-523.

40. Inoue $\mathrm{Y}$, Azuma A, Ogura T et al. All-case post-marketing surveillance of pirfenidone in Japan: clinical characteristics, efficacy and safety profile in 
> 1300 patients with idiopathic pulmonary fibrosis. (IPF). European Respiratory Society Annual Congress 2013; Session 345.

41. Iwasawa T, Ogura T, Sakai $F$ et al. CT analysis of the effect of pirfenidone in patients with idiopathic pulmonary fibrosis. Eur $\mathrm{J}$ Radiol 2012; Mar 30 (Epub ahead of print).

42. Jiang $\mathrm{CH}$, Huang $\mathrm{H}$, Liu $\mathrm{J}$ et al. Adverse events of pirfenidone for the treatment of pulmonary fibrosis: a meta-analysis of randomized controlled trials. PLoS One 7(10): e47024.

** Updated review on pirfenidone's adverse effects.

43. Gahl WA, Brantly M, Troendle $J$ et al. Effect of pirfenidone on the pulmonary fibrosis of Hermansky-Pudlak syndrome. Mol Genet Metab 2002; 76: 234-242.

44. O’Brien K, Troendle J, Gochuico BR et al. Pirfenidone for the treatment of Hermansky-Pudlak syndrome pulmonary fibrosis. Mol Genet Metab 2011: 103: 128-134.

45. InPharm. First idiopathic pulmonary fibrosis drug approved (on line). Available from URL: http://www.inpharm.com/news/149931/intermuneidiopathic-pulmonary-fibrosis-esbriet-pirfenidone (Last accesed 28 August 2013). 
Table 1.- Pirfenidone. Most commonly reported adverse reactions (> 10\% patients) ${ }^{*}$

\begin{tabular}{|l|c|c|}
\hline \multicolumn{1}{|c|}{ Adverse event } & Pirfenidone $2403 \mathrm{mg} /$ day & Placebo \\
\hline Nausea & $32.8^{* *}$ & 13.3 \\
\hline Rash & 28.7 & 8.6 \\
\hline Fatigue & 22.3 & 13.3 \\
\hline Diarrhea & 21.7 & 13.5 \\
\hline Dyspepsia & 16.8 & 5.5 \\
\hline Photosensitivity & 12.2 & 1.7 \\
\hline
\end{tabular}

* Data from clinical studies including 1,345 healthy volunteers and patients (27).

** $\%$ of patients 
Table 2.- Potential drug interactions of pirfenidone

CYP1A2 inhibitors

Fluvoxamine

Ciprofloxacin

Propafenone

Grapefruit juice

CYP2C9 inhibitors

Amiodarone

Fluconazole

Voriconazole

CYP2C19 inhibitors

Chloramphenicol

CYP2D6 inhibitors

Fluoxetine

Paroxetine

Quinidine

CYP1A2 inducers

Tobacco smoke

Omeprazole

Abbreviations: CYP, Cytochrome 


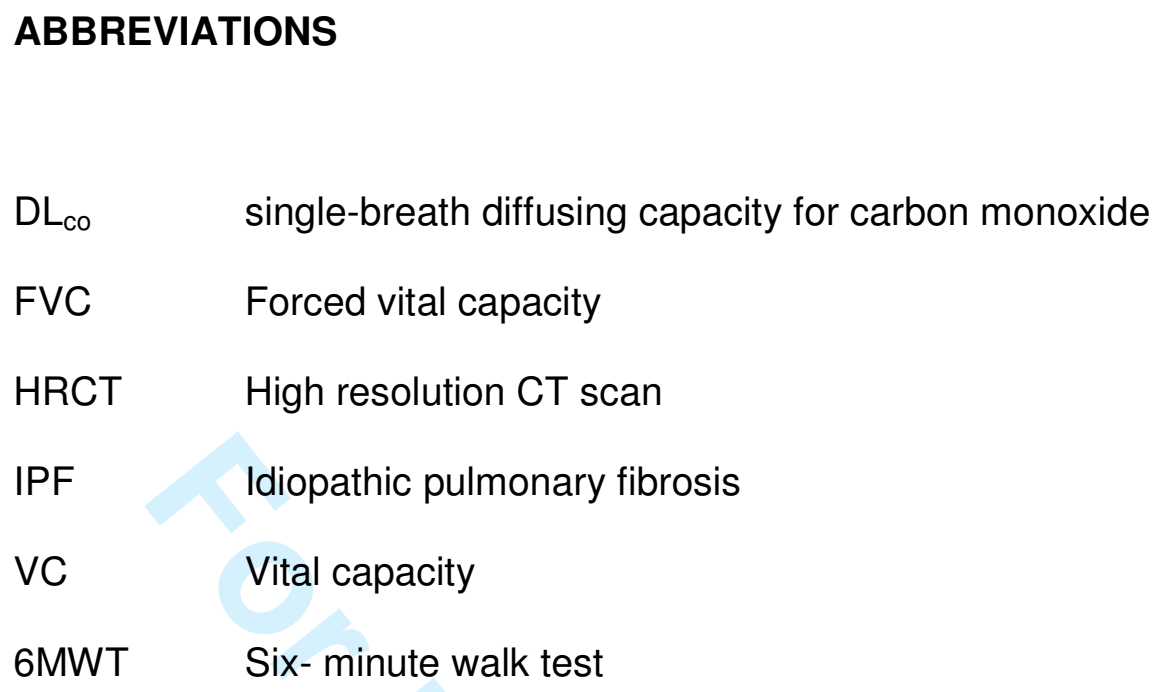

6MWT Six- minute walk test 